\title{
Prediction of parameters affecting the safety of tank farms in case of emergency
}

\author{
Vyacheslav Gorev ${ }^{1, *}$, Vladimir Roytman ${ }^{1}$ \\ ${ }^{1}$ Moscow State University of Civil Engineering, 129337, Moscow, Yaroslavskoye shosse, 26, Russia
}

\begin{abstract}
The current article demonstrates that the physical nature of blow-out of oil products burning in the tank is connected with overheating and fast vaporization of bottom water accumulated in the tank or on a surface of internal floating roof. It is shown that the period of time, during which the homothermal layer of oil, heated up to the boiling point while burning in the tank, reaches the level of bottom water or the water added in the tank in the process of extinguishing depends on the heat losses into environment through the side surface of the tank. It is determined that blow-out time depends on the water cooled surface area of the tank. It is shown that intensive water cooling extremely decreases the rate of formation of homothermal layer with increasing of its thickness.
\end{abstract}

\section{Introduction}

Fires in tanks usually begin with explosion of steam-air mix and disrupt of a roof or damage of integrity of its separate parts [1]. Depending on force of explosion in a vertical metal tank events can develop by several scenarios:

- the roof of tank is damaged, completely disrupted and throwed away on a distance of 20-30 m; liquid burns on all area of the tank;

- the roof of tank is a little raised, completely or partially disrupted, but it is still stay in a burning liquid in semisubmerged condition;

- the roof is deformed in attaching points with the tank wall, small cracks are formed in weld seams of the roof.

In conditions of any of these scenarios fire extinguishing systems of tanks mounted on the top of constructions are unable to work.

In case of combustion in cylindrical horizontal and spherical tanks it is usually disrupt the bottom therefore liquid spreading on a large area creates danger for neighboring tanks and constructions.

Protection of crude oil in drain tank is reached by presence at its bottom of a gas-salt layer of water according to oil production technology.

Displacement of oil from under the ground is made using the water with various additives which flows into the tank together with crude oil. The watery oil supplied for cleaning into the tank where the mixture is stratified with time and oil floats above the water. Water with big concentration of inorganic salts dissolving in it during passing

\footnotetext{
*Corresponding author: ikbs@mgsu.ru
} 
through oil layers, is periodically drained. As a result it is formed two-layer system: oil layer is above, gas-salt layer of water is below. Height of the last can reach 6-8 m.

The physical nature of blow-out of oil products burning in the tank is connected with overheating and fast vaporization of bottom water accumulated in the tank or on a surface of internal floating roof [2].

The period of time, during which the homothermal layer (HTL) of oil, heated up to the boiling point while burning in the tank, reaches the level of bottom water or the water added in the tank in the process of extinguishing, depends on the heat losses into environment through the side surface of the tank [3-5].

\section{Formation homothermal layer}

In order to detect the time of blow-out it is necessary to define the water cooled surface area of the tank [6-8].

Heat flow from a flame jet is spent generally on oil vaporization and formation of a flow of combustible vapors in combustion zone [9-15]. Dispersion of heat through the side area of the metal tank is proportional to a thickness of homothermal layer $-\mathrm{h}$.

Heat spent on vaporization of oil is defined by product of burnup mass rate and combustion value:

$$
Q_{\mathrm{T}}=m Q_{s}+U_{n} F_{o} \rho C\left(T_{n}-T_{o}\right)+\alpha_{1} F_{b}\left(T_{n}-T_{o}\right)+\alpha_{2} F_{o}\left(T_{n}-T_{o}\right)
$$

where: $Q_{\mathrm{T}}-$ flow of heat from a flame jet to a surface of the tank liquid, $\mathrm{J} / \mathrm{s}$;

$m$ - mass burnup rate of a flammable liquid (FL), $\mathrm{kg} / \mathrm{s}$;

$Q_{s}$ - heat value, $\mathrm{J} / \mathrm{Kg}$;

$U_{n}-\mathrm{FL}$ warm-up rate, $\mathrm{m} / \mathrm{s}$;

$F_{o}$ - surface area of FL in the tank, $\mathrm{m}^{2}$;

$\rho-$ FL density, $\mathrm{kg} / \mathrm{m}^{3}$;

$C-$ FL heat capacity, $\mathrm{J}(\mathrm{\kappa g} \cdot \mathrm{K})$;

$T_{n}-\mathrm{FL}$ surface temperature in process of burning, $\mathrm{K}$;

$T_{o}$ - initial temperature of FL equal to the ambient temperature, $\mathrm{K}$;

$\alpha_{1}, \alpha_{2}-$ heat transfer coefficients: through the tank wall and from homothermal layer deep into $\mathrm{FL}, ; \mathrm{J} / \mathrm{m}^{2}(\mathrm{~s} \cdot \mathrm{K})$

$\mathrm{F}_{\mathrm{b}}$ - lateral area of HTL, $\mathrm{m}^{2}$;

The mass burnup rate $m$ we express through the specific $\mathrm{u}_{\mathrm{m}}\left(\mathrm{kg} \cdot\left(\mathrm{m}^{2} \cdot \mathrm{s}\right)\right)$, and lateral area of HTL - through its thickness $h(\mathrm{~m})$ :

$$
\begin{aligned}
& m=u_{m} F_{\mathrm{o}} ; \\
& F_{b}=2 \pi R h,
\end{aligned}
$$

where $R$-radius of the tank, $\mathrm{m}$.

We express $U_{n}$ variable value in an explicit form:

$$
U_{n}=d h / d \tau
$$

The given below figure presents the diagram of the oil tank where the homothermal layer is formed (Figure 1). 
In an equation (1) we transfer time-constant members from the right to the left part and denote so:

$$
\Delta \mathrm{T}=\mathrm{T}_{\mathrm{n}}-\mathrm{T}_{\mathrm{o}}
$$

Substituting the expression (2)-(5) into equation (1), we obtain:

$$
Q_{\mathrm{T}}-u_{m} F_{\mathrm{o}} Q_{\mathrm{c}}-\alpha_{2} F_{\mathrm{o}}\left(T_{\mathrm{n}}-T_{\mathrm{o}}\right)=F_{o} \rho C \Delta T \frac{\mathrm{dh}}{\mathrm{d} \tau}+2 \pi R \alpha_{l} h \Delta T
$$

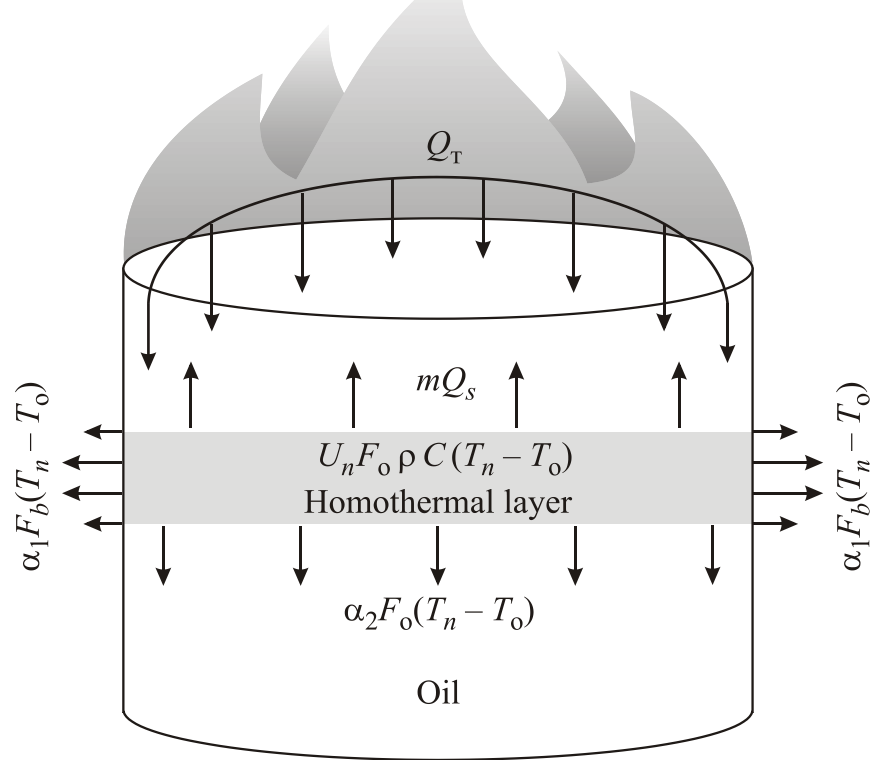

Fig 1. The schema of main heat flows in process of homothermal layer formation in the tank with burning oil product: $\rightarrow$ - main directions of heat flow.

Designating the left part through $q$, we receive an initial differential equation depicting change of thickness of a layer in time:

$$
\begin{aligned}
F_{o} \rho C \Delta T \frac{\mathrm{d} h}{\mathrm{~d} \tau} & =q-2 \pi R \alpha_{1} \Delta T h ; \\
a \frac{\mathrm{d} h}{\mathrm{~d} \tau} & =q-b h,
\end{aligned}
$$

where: $a=F_{\mathrm{o}} \rho C \Delta T ; b=2 \pi R \alpha_{1} \Delta T$.

The solution of equation (8) with the initial conditions of $\tau=0$ and $h=0$ is the correlation:

$$
\tau=\frac{a}{b} \ln \frac{q}{q-b h}
$$


Let's enter the designation:

$$
\tau_{0}=\frac{a}{b}=\frac{\rho c R}{2 \alpha_{1}}
$$

Let's remove the $h$ value from the equation (9):

$$
h=\frac{q}{b}\left(1-e^{-\tau(b / a)}\right)
$$

In conditions when $\tau \rightarrow \infty$ the $h$ value becomes maximal

$$
h h_{m}=a / b,
$$

where: $h_{m}$ - maximum possible thickness of the homothermal layer, m.

Let's find $d h / d \tau$ derivative:

$$
-\left(\frac{\mathrm{d} h}{\mathrm{~d} \tau}\right) \equiv U_{n} \equiv \frac{q}{a} e^{-\tau(b / a)}
$$

If $\tau \rightarrow 0$, than:

$$
U_{n}=U_{0} \equiv \frac{q}{a}
$$

where $U o$ - initial warm-up rate of liquid, $\mathrm{m} / \mathrm{s}$.

From the correlation (14) we shall receive the formula for calculation of $q$ value:

$$
q=a U_{0}
$$

If the fraction under the logarithm in the formula (9) will be divided by $b$ after replacing $q$ by $a U_{0}$ from formula (15), we shall receive:

$$
\tau=\tau_{0} \ln \frac{\tau_{0} U_{0}}{\tau_{0} U_{0}-h}=\tau_{0} \ln \frac{h_{m}}{h_{m}-h}
$$

where:

$$
h_{m}=\tau_{0} U_{0} \equiv U_{0} \frac{a}{b} \equiv \frac{q}{b}
$$

$\tau_{0}$ - starting time of liquid warm-up, $\mathrm{h}$.

We shall similarly simplify the formula (11)

$$
h=h_{m}\left(1-e^{-\tau(b / a)}\right)
$$


Formulas (16) and (18) describe dynamics of change of homothermal layer thickness and can be used for prediction of oil product blow-out time during long-continued fire. The applicability of the (16) correlation is limited by condition of $h_{m}>>h$, that is the maximal possible thickness of homothermal layer should be must be appreciably greater than the thickness of a layer of fuel in the tank.

Numerical values of the $\alpha_{1}$ heat-transfer coefficient on the "tank wall - air" boundary can be defined from criteria correlation for free turbulized convection near the heated wall [3]:

$$
\mathrm{Nu}=0.1(\mathrm{Gr} \cdot \mathrm{Pr})^{1 / 3}
$$

where: $\mathrm{Nu}$ — Nusselt number; Gr — Grashof number; Pr — Prandtl number.

In our case $\operatorname{Pr}=0.75$ [3], consequently:

$$
1=0.09 \lambda\left(\frac{\beta}{v}\right)^{\frac{1}{3}}
$$

where: $\lambda$. - heat conductivity coefficient of heat-transfer agent, $\mathrm{W} /(\mathrm{m} \cdot \mathrm{K})$;

$\beta-$ coefficient of thermal expansion, $\mathrm{K}^{-1}$;

$v$ - kinematic coefficient of viscosity, $\mathrm{m}^{2} / \mathrm{s}$.

If surface of the tank is cooling by water heat-transfer coefficient can be defined by this correlation:

$$
\mathrm{Nu}_{2}=0.25 \mathrm{Ar}^{1 / 3}
$$

where $\mathrm{Ar}$ - Archimedean number.

This correlation allow for a mode of film evaporation of water in case of contact with heated surface of the tank:

$$
\alpha_{1}=0.25 \frac{\lambda_{f}}{v_{f}} g^{1 / 3}
$$

where:

$\lambda_{f}$ - heat conductivity coefficient of a film, $\mathrm{W} /(\mathrm{m} \cdot \mathrm{K})$;

$v_{f}$ - kinematic coefficient of viscosity of a film, $\mathrm{m}^{2} / \mathrm{s}$;

$g$ - free fall acceleration, $\mathrm{m} / \mathrm{s}^{2}$.

If the cooling is conducted with a small consumption of water average value of $\alpha$ can be reduced in proportion to watered surface.

In case of intensive water cooling the speed of homothermal layer formation is extremely reduced with the growth of its thickness. The maximal thickness of homothermal layer is determined by correlation of heat quantity going out through the tank walls which increases in process of warming-up the liquid and value of heat flow from the flame jet to the combustion surface.

If the maximal thickness of homothermal layer is less than distance from its surface to the aqueous layer at the bottom of the tank than the time through which the homothermal layer reaches the water will be determined by warming-up rate as well as by burn-out rate of oil product.

Let's output the calculated correlation for the case, when the maximal thickness of homothermal layer is less than flammable liquid $\mathrm{h}_{\mathrm{g}}$, i.e. $h_{m}<h_{\mathrm{g}}$ (the $h_{m}$ value is calculated by (17) formula). 
In the general case the lower bound of homothermal layer will be determined as by warming-up of the flammable liquid so by lowering of its level in process of burnout. If as the reference point will be taken the level of liquid before combustion than coordinate of the lower boundary of homothermal layer can be defined by such correlation:

$$
z=U_{1} \tau+U_{n} \tau
$$

where $U_{1}$ - linear rate of burn-out, $\mathrm{m} / \mathrm{s}$.

We express $U_{n}$ value in an explicit form:

$$
z=U_{1} \tau+\frac{q}{b}\left(1-e^{-\tau(a / b)}\right)
$$

If $z>>h_{m}$ then value $e^{-\tau(a / b)}$ is negligible. So we can get such example:

$$
z=U_{1} \tau+\frac{q}{b}=h
$$

Taking into account that $q=a U_{0}$ and having the $\alpha$ and $b$ values we shall receive an equal determining the time from the beginning of warm-up to the blow-up of FL, if $h_{m}<<$ $h_{\mathrm{g}}$ :

$$
\tau=\frac{h_{g}}{U_{1}}-\frac{U_{0}}{U_{1}} \frac{\rho R}{2 \alpha}=\frac{h_{g}-\tau_{0} U_{0}}{U_{1}}
$$

To define the time of blow-out the oil product we use two correlations:

if $h_{m} \gg h_{g}$ or $\tau_{0} U_{0} \gg h_{g}$

$$
\tau=\tau_{0} \ln \frac{\tau_{0} U_{0}}{\tau_{0} U_{0}-h_{g}}
$$

where

$$
\tau_{0}=\rho C R /(2 \alpha)
$$

if $h_{m}<<h_{g}$

$$
\tau=\frac{h_{g}-h_{m}}{U_{1}}
$$

From the obtained correlations we can see that warming-up value of liquid, its linear burn-out rate, initial level of flammable liquid in tank and the thickness of homothermal layer have an impact on the blowing-up time of the oil product burning in tank. 


\section{Determinig the thickness of homothermal layer}

For determination of the thickness of homothermal layer at the set time point $(h)$ it is possible to use two variants of formulas:

From the ratio (27) we omit intermediate transformations:

If $h_{m}>>h_{g}$, than we receive

$$
h=\tau_{0} U_{0}\left(1-e^{-\tau / \tau_{0}}\right)
$$

From the ratio (23)

if $h_{m}<<h_{g}$, than we receive

$$
z=U_{1} \tau+U_{0} \tau_{0}
$$

Accordingly, distance to water layer $\delta$ at the bottom of the tank at the set timepoint (if $h_{m}<<h_{g}$ ) is determined by formula:

$$
\delta=h-z=h-\left(U_{1} \tau+U_{0} \tau_{0}\right)
$$

Let's define the time before blow-up of oil product (gasoline) in case of fire in partially water-cooled tank by substituting in a formula (27) the following parameters [4]: $R=26 \mathrm{~m}$, $\rho=700 \mathrm{~kg} / \mathrm{m}^{3}, C=2 \cdot 10^{3} \mathrm{~J} /(\mathrm{Kg} \cdot \mathrm{K})$, initial level of FL in tank $h_{\mathrm{g}}=9.5 \mathrm{~m}$, the thickness of water layer under the FL- $0,5 \mathrm{M}$, burn-out velocity of gasoline $U_{1}=0.4 \mathrm{M} / \mathrm{u}$, initial time warming-up rate $U_{0}=1.8 \cdot 10^{-4} \mathrm{~m} / \mathrm{s}$, heat transfer coefficient in conditions of free combustion $\alpha_{1}=10 \mathrm{~W} /(\mathrm{m} \cdot \mathrm{K}), \alpha_{2}=730 \mathrm{~W} /(\mathrm{m} \cdot \mathrm{K})$.

To select the calculated correlations let's define the $h_{m}$ value:

$$
\begin{aligned}
& \tau_{02}=700 \cdot 2 \cdot 10^{3} \cdot 13 /(2 \cdot 730)=12.5 \cdot 10^{3} \mathrm{~s} \\
& h_{m 2}=12.5 \cdot 10^{3} \cdot 1.8 \cdot 10^{-4}=2.2 \mathrm{~m} .
\end{aligned}
$$

Consequently, $h_{m}<<h_{\mathrm{g}}$, therefore for the calculation we use the formula (29):

$$
\tau_{2}=\frac{h_{g}-h_{m}}{U_{1}}=\frac{9.5-0.5}{0.4}=\frac{9}{0.4}=22.5 h
$$

\section{Conclusion}

Thus, in considered fire conditions the blow-out of oil product will occur after 22.5 hours, so the fire should be localized and liquidated during the day.

So, as a result of the conducted analysis and calculations it was formulated the prognosis of a time period during which the homothermal layer (HTL) reaches the level of the tank bottom water or water supplied into the tank during fire suppression.

Based on analysis of heat flows coming from flame to a burning oil surface and dispersing into environment it was deduced the calculated ratio of dependence of thickness of the homothermal layer on the burning time of oil in the tank.

The intensive water cooling is sharply decreasing the speed of formation of homothermal layer together with increase of its thickness. The maximal thickness of homothermal layer is determined by ratio of heat losses which increase in process of 
warming-up of a liquid and heat flow from the flame jet to a burning surface.

If the maximal thickness of homothermal layer is less than the distance from the surface to water layer located at the bottom of the tank, than the time during which the homothermal layer reaches the water lavel will be determined not only by speed of warming-up but also by burn-out rate.

\section{References}

1. P. Nash, D. Hird, R. J. French, Base Injection of Foam for Fuel Storage Tanks, (1960)

2. V. I. Blinov, G. N. Khudyakov, Diffusive burning of liquids. Academy of Sciences of the USSR, (1961)

3. V. I. Blinov, G. N. Khudyakov, I. I. Petrov, V. Ch. Reutt, On movement of the tank liquid while mixturing by the air stream. Mechanism of flame suppression in oil tanks.

Prod. Ministry of Housing and Communal Services of RSFSR, (1958)

4. W. H. MacAdams, Heat transfer. Translation from engl. vers. Leningrad, (1936)

5. H. S. Mahley, Mobil Oil Corporation, (1967)

6. S. A. Sharovarnikov, D. A. Korolchenko, A. V. Lyapin, Fire and Explosion Safety 23(6), 76-80 (2014)

7. B. Persson, A. Lönnermark, H. Persson, D. Mulligan, A. Lancia, M. Demichela, FOAMSPEX - Large Scale Foam Application - Modelling of Foam Spread and Extinguishment, SP Swedish National Testing and Research Institute, SP Report 2001:13, (2001)

8. D. A. Korolchenko, A. F. Sharovarnikov, Fire and Explosion Safety 23(11), 84-88 (2014)

9. D. A. Korolchenko, S. A. Sharovarnikov, A.V. Byakov, Advanced Materials Research 1073-1076, 2353-2357 (2015)

10. D. Korolchenko, A. Tusnin, S. Trushina, A. Korolchenko, International Journal of Applied Engineering Research 10(21), 42541-42548 (2015)

11. A. F. Sharovarnikov, D. A. Korolchenko, Applied Mechanics and Materials 475-476, 1344-1350 (2014)

12. I. F. Bezrodnyi, Fire and explosion safety 12, 49-55 (2011)

13. S. Korzeniowski, T. Cortina, Industrial Fire Journal, 18-20 (2008)

14. Q. Zhang, L. Wang, Y. Bi, H. Zhi, P. Qiu, Journal of Hazardous Materials 287, 87-92 (2015)

15. H. Ranjbar, B. H. Shahraki, Chemical Engineering and Technology 36, 295-299 (2013) 